\title{
Perception of the Laser Radiation for the Near Infrared Range
}

\author{
D. $\operatorname{KECIK}^{a}$, J. KASPRZAK ${ }^{a, *}$ AND A. ZAJĄC ${ }^{b}$ \\ ${ }^{a}$ Department and Ophthalmology Clinic, I Medicine Faculty, Medical University of Warsaw \\ W. Lindleya 4, 02-005 Warsaw, Poland \\ ${ }^{b}$ Institute of Optoelectronics, Military University of Technology, S. Kaliskiego 2, 00-908 Warszawa, Poland \\ During the diagnostic research done by means of optical devices equipped with radiation sources from the near \\ infrared range the phenomena indicating the perception possibility of this range by a human eye were observed. \\ In this contribution the initial results of the research of this phenomenon were presented. Sources of radiation \\ applied in laser polarimeters $(785 \mathrm{~nm})$ and devices designed for optical coherent tomography $(820,850 \mathrm{~nm})$ were \\ taken into particular consideration. Perception tests with the use of a laser diode generating at the wavelength \\ of $940 \mathrm{~nm}$ were also carried out. It was stated that the radiation from the range examined can be recorded by a \\ human eye giving a colour sensation - in practice independently of the wavelength of the radiation beam falling \\ into a retina.
}

PACS: 42.66.-p, 42.66.Ct, 42.66.Ne

\section{Introduction}

The spectral range of the visible radiation is not clear-cut defined. The boundary values depend on the individual sensitivity of the observer eye and the energetic value of the flux reaching a retina. According to the EIC standard it is assumed that the boundary values of the human eye perception range equal from $380 \mathrm{~nm}$ to $780 \mathrm{~nm}$. During the metrological verification of the optical coherent tomography system (OCT1) made by Humphrey it was stated that the diagnostic beam scanning the eyeground causes (at a patient) the so-called optokinetic nystagmus. This phenomenon consists in the instinctive tracking of the diagnostic beam during the eyeground scanning [1].

In the device discussed the light-emitting diode (LED) generating $750 \mu \mathrm{W}$ power at the wavelength of $850 \mathrm{~nm}$ is applied. The time duration of a single scan equals about $1.5 \mathrm{~s}$. Although the wavelength of the LED radiation lies beyond the visual range, the patient sees the diagnostic beam as the orange-and-red spot moving in accordance with the scanning beam movement. The involuntary eye motions caused by the diagnostic beam movement make the fixation impossible and they are the source of the artefacts deforming the results of the tomographic examination. For the sake of the considerable inconvenience, the phenomenon discussed has been analyzed in detail.

\section{Examination methodology}

In the group of 150 patients (95 women and 55 men aged between 20 and 75 years old) referred to the OCT

* corresponding author; e-mail: kasprzak@amwaw.edu.pl laboratory, the perception tests of the diagnostic beam were carried out. During the test the patient described the spot location, the speed and the direction of the diagnostic beam movement within the rear eyeball pole (during tomogram recording). The power of the diagnostic beam was controlled in the range of $10-750 \mu \mathrm{W}$. The linear, circular, rectangular scans and the scans crossing at an angle were used. The device allowed us using the two speeds of the beam movement. Faster diagnostic beam speed was used during the examination of the scan localization while slower diagnostic beam speed was used for tomogram recording. In the first case the patient's eye can record the beam as a line characterized by a specific shape, however, in the other case as a point moving along the suitable contour. The eyeground was lightened by means of the device equipped with the halogen illuminator. This device allowed us controlling the light intensity - with the use of the red filter. The perception tests were repeated in a group of 40 people referred to the examination carried out by means of OCT STRATUS system (a diagnostic source generating at the wavelength of $820 \mathrm{~nm})$.

\section{Results}

During the clinical examinations it was stated that when the illuminator was on, all the patients saw the diagnostic beam in the accessible region of the eyeball rear pole in full range of the control of the beam power (provided that the retina in the area examined was not damaged). According to the expectations the beam perception decreased within the ophthalmic nerve disk. When the diagnostic beam intensity was maximum, the illumination of the eyeground through the red filter did not block the perception in the accessible perception range. 
The illuminating of the eyeground by means of the white light beam made the perception impossible at the beam intensity dazzling the patient (the low perception of the green, fixation point located in the patient visual field was observed). The decrease of the diagnostic beam intensity allowed us determining the perception threshold at a given radiation intensity within the eyeground. When searching the causes of the perception of a diagnostic beam it was assumed that the radiation from the visible range generated within the measuring system is the subject to the perception. In order to verify this hypothesis, an attempt at cutting off the visual range was done locating the 03FCG118 filter (made by Melles Griot) before the objective of the OCT1 head. The filter characteristic is presented in Fig. 1. This filter attenuated effectively in the visible range. The transmission in the long-wave range of the visible range changes from 0 (for $760 \mathrm{~nm}$ ) to 0.0015 (for $780 \mathrm{~nm}$ ). In spite of this, after locating the filter on the diagnostic beam path the beam perception recorded by a patient practically does not change (the patients reported an insignificant decrease in the spot intensity).

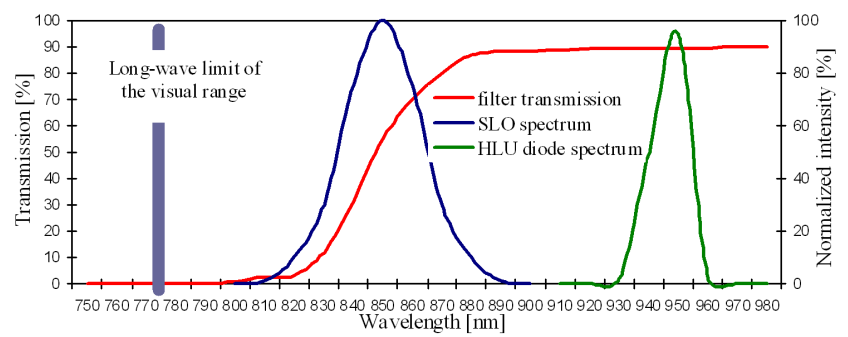

Fig. 1. The spectrum of the superluminescent diode (SLD) used in OCT1 system and the spectrum of the laser diode (HLU 25F) against the background of the transmission filter (03FCG114) used in order to cut off the visual range of the radiation.

A similar phenomenon of an atypical perception can be noticed in case of the observation of the diagnostic beam for the analyzer of nerve fibers (GDx). In this device the radiation of a diode laser emitting at the wavelength of $785 \mathrm{~nm}$ is used. Also in this case the patients record the diagnostic beam image. As a result of definitely higher scanning frequency, the patient observes the yellow-red lines filling the accessible visual field.

The phenomenon of an atypical perception of the laser radiation can be observed in case of other sources from the range of near infrared. The perception range evaluation was expanded using a laser diode (HLU 25F 400$-940 \mathrm{P})$ generating continuous power up to $0.8 \mathrm{~mW}$ at the wavelength of $940 \mathrm{~nm}$. Tests (on three patients) done in laboratory conditions confirmed the appearance of the perception phenomenon for this radiation range. The characteristic of this perception type is the colour of the spot observed - practically independently of the source wavelength.

\section{Summary}

In literature, the phenomenon described above is explained as the approximation of the eye sensitivity model used for the near infrared range $[2,3]$. For the wavelength of $850 \mathrm{~nm}$ the eye sensitivity evaluated equals $1.1 \times 10^{-7}$ while for the wavelength above $945 \mathrm{~nm}$ it equals $3.2 \times 10^{-9}$. Therefore, in case of radiation characterized by the wavelengths above $900 \mathrm{~nm}$ it is necessary (to reach the perception threshold) to use the radiation intensities dangerous for an eye.

The perception of the diagnostic beam of the wavelength of $850 \mathrm{~nm}$ was confirmed in our own experiments — even below $10 \mu \mathrm{W}$. Also, a very interesting aspect is the specific colour observed during the observation process of the radiation from this range. The phenomenon observed shows that in case of the radiation sources analyzed the real biochemical processes occurring in the retina receptors do not have to agree with the up-to-date knowledge. The detailed analysis of this phenomenon requires initiating the new research. The results of this research probably will become the source of important information in the area of eye physiology. They may also affect methods of the safety evaluation of laser sources and make possible the development of new diagnostic methods.

\section{References}

[1] J. Kasprzak, Ph.D. Thesis, Military University of Technology, Warsaw 2004 (in Polish).

[2] A. Stockman, L.T. Sharpe, C. Fach, Vis. Res. 39, 2901 (1999)

[3] A. Stockman, L.T. Sharpe, Vis. Res. 40, 1711 (2000). 